\title{
Erythema multiforme associated with Mycoplasma pneumoniae
}

\author{
J. A. Vargas-Hitos • M. V. Manzano-Gamero • \\ J. Jiménez-Alonso
}

Received: 29 December 2013/Accepted: 31 March 2014/Published online: 12 April 2014

(C) Springer-Verlag Berlin Heidelberg 2014

Fig. 1 Vesiculopustular exudative lesions over the face and vesicular "target" lesions in the neck and the hands highly suggestive of erythema multiforme
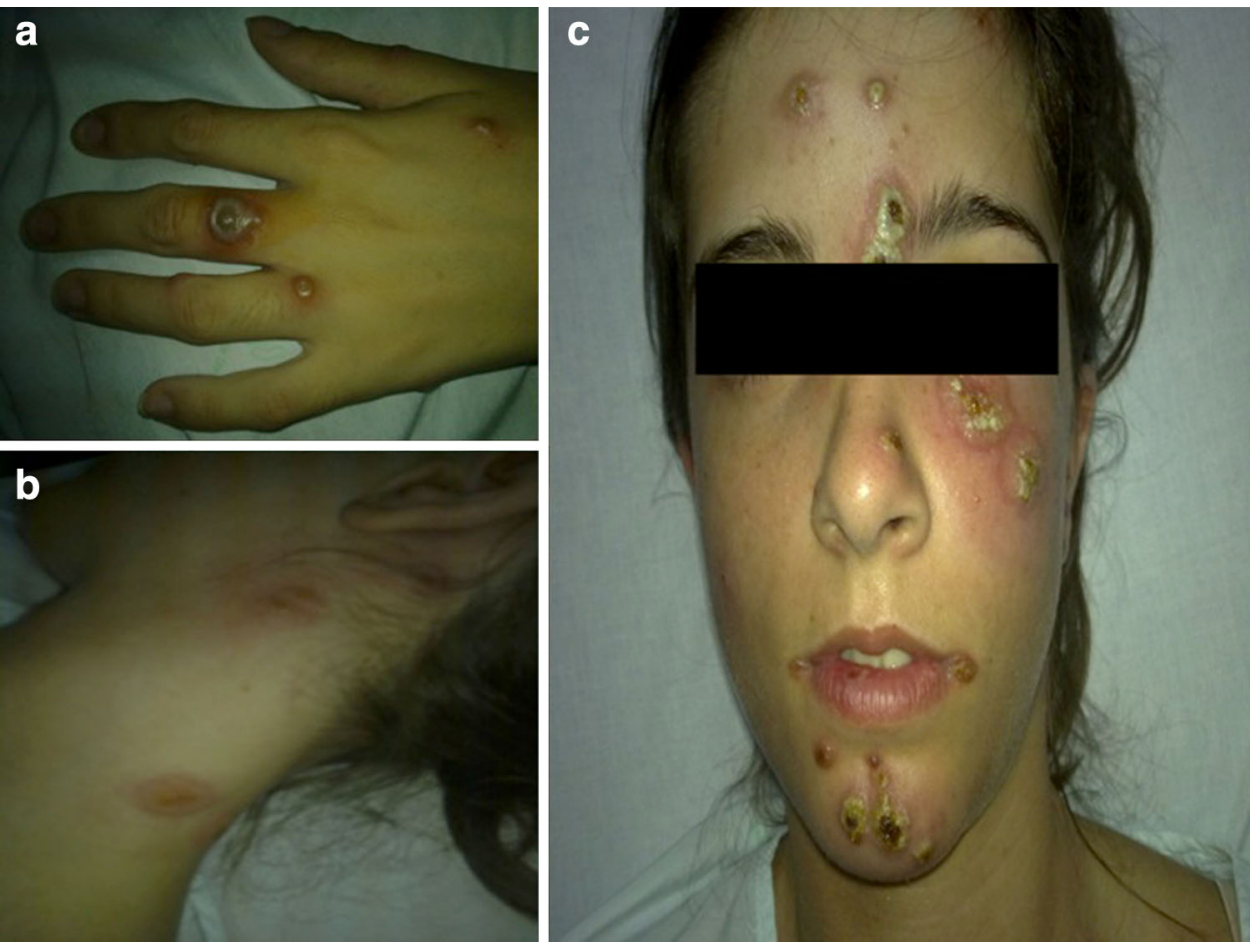

A 21-year-old-woman with no relevant medical history presented with a 1-week history of sore throat, fever and itchy rash. The examination revealed erythema in the oropharynx and vesiculopustular exudative lesions $2-3 \mathrm{~cm}$ in diameter over the face and erythematous vesicular

J. A. Vargas-Hitos $(\bowtie) \cdot$ M. V. Manzano-Gamero .

J. Jiménez-Alonso

Internal Medicine Department, Virgen de las Nieves University

Hospital, 9th floor, Avda. Fuerzas Armadas $\mathrm{N}^{\circ} 2$,

18014 Granada, Spain

e-mail: joseantoniovh@hotmail.com "target" lesions in the trunk, upper limbs and the back of both hands (Fig. 1a-c). Laboratory tests, with the exception of the C-reactive protein level $(4 \mathrm{mg} / \mathrm{dL})$, were all normal. Blood cultures and cultures from skin lesions (for both virus and bacteria) were negative. However, the serological test for Mycoplasma pneumoniae (IgM; microparticle agglutination assay) was positive. Treatment with intravenous levofloxacin and topical mupirocin was initiated and the skin lesions gradually improved and the fever disappeared. A biopsy of the lesions was not performed given the improvement of the patient with the 
treatment administered and the positivity of the serology. However, a second blood sample was obtained 10 days afer the first serology result, observing a four-fold increase in IgM titre and, thus, the diagnosis of erythema multiforme (EM) secondary to M. pneumoniae infection could be made.

M. pneumoniae can cause skin lesions in up to $25 \%$ of patients with pulmonary infections [1]. A non-specific exanthem is the most common cutaneous reaction related to $M$. pneumoniae, but other skin conditions, such as pityriasis rosea, Kawasaki disease, leukocytoclastic vasculitis, subcorneal pustular dermatosis, Sweet syndrome, toxic epidermal necrolysis, Henoch-Schönlein purpura and EM have been previously described [2]. However, the aetiologies of EM are commonly drugs (antibiotics, anticonvulsants) and infections (herpes simplex virus mainly). Since $M$. pneumoniae is a frequent cause of respiratory tract infection, being aware of potential mucocutaneous manifestations in these patients is advisable to any physician.
On the other hand, patients presenting with EM should be evaluated for, but not only, herpes simplex virus, since many others infectious agents, such as M. pneumoniae, may produce it.

Conflict of interest On behalf of all authors, the corresponding author states that there is no conflict of interest.

Ethical standards This study was approved by the local ethics committee.

\section{References}

1. Murray HW, Masur H, Senterfit LB, Roberts RB. The protean manifestations of Mycoplasma pneumoniae infection in adults. Am J Med. 1975;58:229-42.

2. Schalock PC, Dinulos JG, Pace N, Schwarzenberger K, Wenger JK. Erythema multiforme due to Mycoplasma pneumoniae infection in two children. Pediatr Dermatol. 2006;23:546-55. 